\title{
Enterovirus Gastroenteritis
}

National Cancer Institute

\section{Source}

National Cancer Institute. Enterovirus Gastroenteritis. NCI Thesaurus. Code C35624.

Gastroenteritis resulting from an infection with enterovirus. 